\title{
Impactación cubital. Procedimiento artroscópico de Wafer
}

\author{
Ulnar impaction. Wafer arthroscopy procedure \\ René Ochoa Cázares, * José Antonio Chávez Sevilla \\ Citar como: Ochoa CR, Chávez SJA. Impactación cubital. Procedimiento artroscópico de Wafer. \\ Acta Med GA. 2022; 20 (1): 91-95. https://dx.doi.org/10.35366/103565
}

\section{Resumen}

El procedimiento de Wafer es un tratamiento eficaz para el síndrome de impactación cubital, que descomprime la unión cubitocarpiana mediante un abordaje artroscópico o abierto limitado. En comparación con otros procedimientos descompresivos comunes, el procedimiento de Wafer no requiere que exista consolidación ósea o fijación interna y también proporciona una excelente exposición de la superficie proximal del complejo de fibrocartílago triangular. Los resultados del procedimiento de Wafer han sido buenos y se han informado pocas complicaciones. Presentamos el caso de paciente femenina con diagnóstico de impactación cubital, en la cual el tratamiento médico conservador ha fallado y se realizó procedimiento artroscópico de Wafer con excelentes resultados clínicos.

Palabras clave: Impactación cubital, complejo fibrocartílago triangular, artroscopia de muñeca, osteotomía de acortamiento cubital.

\section{INTRODUCCIÓN}

El síndrome de impactación cubital es causado cuando el complejo fibrocartílago triangular es comprimido patológicamente entre una varianza cubital distal positiva o dinámica y el semilunar o piramidal. El síndrome de impactación cubital, el cual es un problema radiocubital distal, está asociado a ruptura degenerativa del complejo fibrocartílago triangular, condromalacia del semilunar, ruptura o atenuación del ligamento lunopiramidal. ${ }^{1}$

\section{Abstract}

The Wafer procedure is an effective treatment for ulnar impaction syndrome, which decompresses the ulnocarpal junction through a limited open or arthroscopic approach. In comparison with other common decompressive procedures, the wafer procedure does not require bone healing or internal fixation and also provides excellent exposure of the proximal surface of the triangular fibrocartilage complex. Results of the wafer procedure have been good and few complications have been reported. We present the case of a female patient with a diagnosis of ulnar impaction in whom conservative medical treatment has failed and a Wafer arthroscopic procedure was performed with excellent clinical results.

Keywords: Ulnar impaction, triangular fibrocartilage complex, wrist arthroscopy, ulnar shortening osteotomy.

El complejo fibrocartílago triangular (CFCT) debe su nombre a su composición histológica: fascículos fibrosos a los que se añaden elementos cartilaginosos en porción variable. Es una estructura ligamentaria fibrocartilaginosa. Se extiende horizontalmente entre la cabeza del cúbito y la primera fila del carpo en su borde cubital. ${ }^{2}$

Funcionalmente el CFCT actúa como un importante estabilizador de la articulación radiocubital distal (RCD), transmite carga axial entre el carpo y el cúbito, también estabiliza el aspecto cubital del carpo, limita la desviación
* Director del Curso Artroscopia y Reconstrucción Articular.

* Fellow en Artroscopia y Reconstrucción Articular.

Hospital Angeles Pedregal. Facultad Mexicana de Medicina de la Universidad La Salle Campus México. México.
Correspondencia:

Dr. René Ochoa Cázares

Correo electrónico: roc.ortopedia@gmail.com

Aceptado: 14-04-2021.

www.medigraphic.com/actamedica 
cubital evitando el choque entre el piramidal y la cabeza del cúbito, manteniendo así un necesario espacio entre el carpo y el cúbito. ${ }^{2}$

El síndrome de impactación cúbito-carpiano se observa en pacientes con un cúbito plus congénito, también como consecuencia de una mala unión postfractura del radio, en la deformidad de Madelung, puede presentarse en secuela de trauma que afectó el crecimiento de la fisis del radio, y en lesiones de codo como Essex-Lopresti. ${ }^{2}$

Palmer y Werner han demostrado que el cúbito neutro en la articulación de la muñeca transfiere el $20 \%$ de la carga axial del antebrazo, cuando la varianza cubital es incrementada a $2.5 \mathrm{~mm}$, la carga axial del antebrazo en el cúbito aumenta 40\%; contrariamente cuando la varianza cubital decrece a menos $2.5 \mathrm{~mm}$, la carga axial del antebrazo ejercida hacia el cúbito decrece en 5\%.3,4 Los procedimientos que le quitan carga a la articulación cubitocarpiana son beneficiosos, ya que alivian los síntomas mecánicos y la irritación que no son aliviados solo por el desbridamiento del complejo fibrocartílago triangular. ${ }^{1}$

En 1941 Milch llevó a cabo la osteotomía de acortamiento cubital para el síndrome de impactación cubital, llevando al paciente al alivio de los síntomas. ${ }^{5}$ Desde entonces el principio del tratamiento quirúrgico para el síndrome es quitar carga a la articulación cubitocarpiana. ${ }^{6}$ Feldon y su grupo en 1992 describieron la osteotomía de Wafer para el cúbito distal. ${ }^{7}$ Subsecuentemente, Wnorowski y sus colegas describieron el procedimiento quirúrgico de osteotomía de Wafer asistido artroscópicamente. ${ }^{8}$ Estas opciones quirúrgicas han sido comúnmente usadas para tratar el problema de impactación cubital. ${ }^{6}$

La varianza cubital positiva puede aparecer:

1. En una posición congénita de la superficie articular del cúbito más distal que la del radio.

2. Adquirida por un acortamiento del radio secundario (unión defectuosa o acortamiento tras fracturas de radio distal, o migración proximal tras la exéresis de la cabeza del radio).

3. Con excesiva variación ulnar dinámica. Mayor traslación distal de la cabeza cubital durante la desviación cubital, la pronación y la prensión.

El síndrome de impactación cubital no es exclusivo de las muñecas con varianza cubital positiva, sino que puede aparecer en muñecas con varianza cubital neutra o incluso negativa. Se debe a que la varianza puede incrementarse durante diferentes actividades, especialmente aquéllas que implican pronación del antebrazo y prensión. ${ }^{9}$

La clasificación de Palmer (1989) de las lesiones del CFCT tipo II describe el proceso degenerativo de la impactación cubital, en ocasiones los estadios pueden superponerse.

El conocimiento de la clasificación y el reconocimiento de las lesiones nos ayudarán a escoger el tratamiento más adecuado en cada caso.

1. Desgaste del CFCT.

2. Desgaste del CFCT con condromalacia del semilunar y/o la cabeza cubital.

3. Perforación del CFCT con condromalacia del semilunar y/o la cabeza cubital.

4. Perforación del CFCT con condromalacia del semilunar y/o la cabeza cubital más perforación del ligamento lunopiramidal.

5. Perforación del CFCT con condromalacia del semilunar y/o la cabeza cubital más perforación del ligamento lunopiramidal más artritis ulnocarpiana. ${ }^{10}$

\section{Presentación de caso}

Se trata de mujer de 50 años, sin antecedentes personales patológicos, con sobrepeso por índice de masa corporal de 28 , sin historia de trauma reciente, no practica ningún deporte, fumadora ocasional.

Refiere sintomatología de dolor en el borde cubital de la muñeca derecha EVA 7/10, incluso ya después de haberse manejado el dolor con analgésicos y con órtesis de inmovilización de la muñeca derecha por aproximadamente cinco meses.

En exploración física, la queja principal de la paciente fue dolor en la muñeca derecha, en especial del lado cubital de la misma, no enrojecimiento, no tumefacción o equimosis al momento de la exploración, no hay chasquido doloroso en la muñeca, la prueba de estrés ulnocarpal, que consiste en realizar movimiento suave de pronaciónsupinación, con la muñeca en máxima desviación cubital y aplicando carga axial a la muñeca despertó dolor en la paciente (test de Nakamura 1997).

Se le pidió a la paciente que se levante de la silla apoyada en sus manos y empujando, maniobra que despertó dolor (maniobra de presión positiva). Se midió por goniometría los arcos de movilidad de la muñeca derecha resultando: flexión 0-90, extensión 0-70, aducción 0-50, abducción 0-20, encontrando leve limitación del arco de movilidad de aducción de la muñeca derecha en comparación con la izquierda.

Luego de estudios radiológicos, se observa una varianza cubital neutral, la epífisis distal y apófisis estiloides cubital presentan cambios quísticos erosivos subcondrales con edema medular reactivo de distribución difusa, sin fracturas o fisuras, siguiendo el eje de la superficie articular. Imagen similar, pero en menor grado en el borde proximal dorsal 
Figura 1:

Imágenes de resonancia magnética de la muñeca derecha.

A) Imagen coronal potenciada en densidad de protones con fat sat, se muestra (flecha blanca) perforación central de FCT. B) Imagen coronal potenciada en densidad de protones con fat sat, se muestra (flecha blanca discontinua) erosión condral de la porción cubital semilunar. C) Imagen coronal potenciada en

T1, se muestra (flecha negra discontinua) erosión condral de la porción cubital del semilunar.

D) Imagen coronal potenciada en densidad de protones con fat

sat, se muestra (flecha blanca discontinua) comunicación entre las articulaciones cubitocarpiana y radiocubital distal.
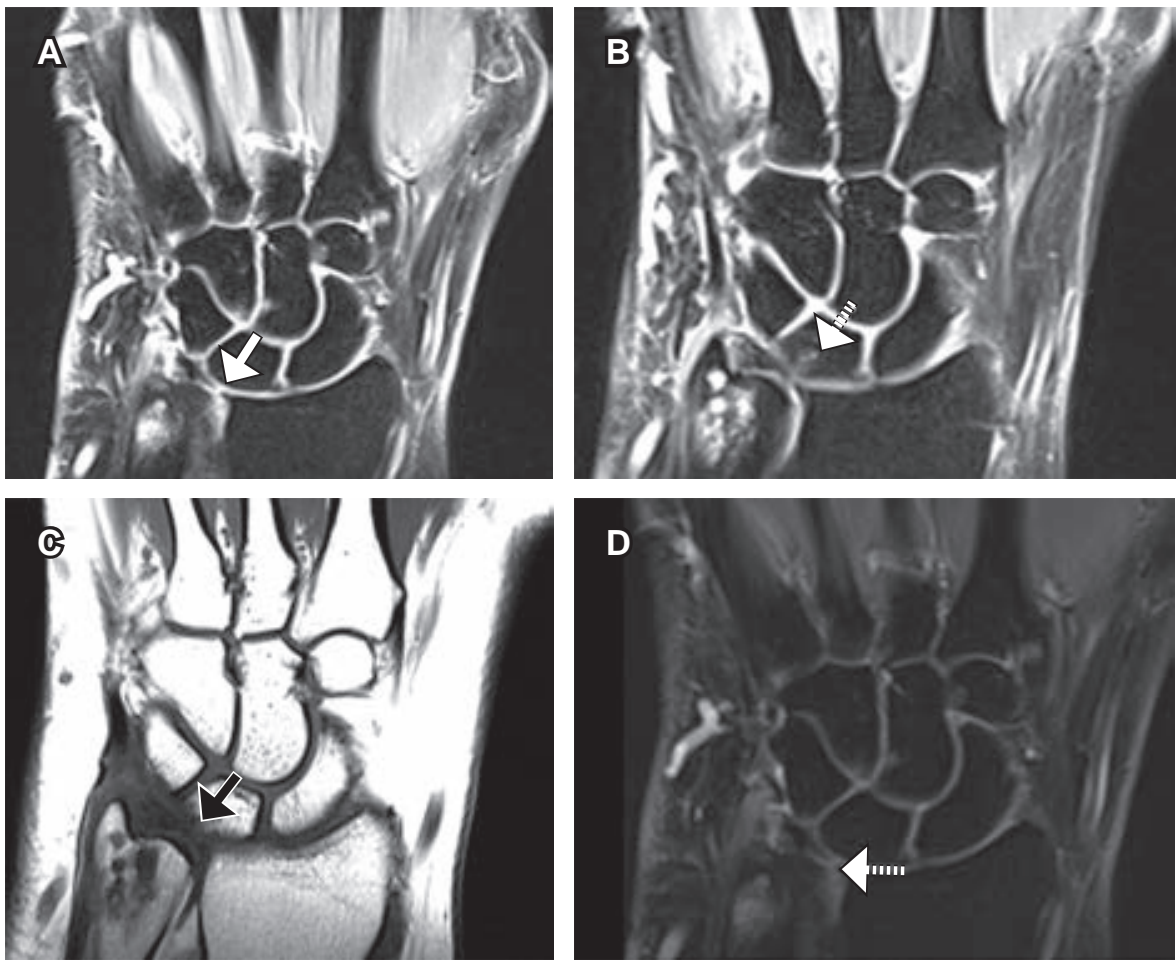

del semilunar, leve edema difuso del fibrocartílago triangular, sin desgarro ni desinserción (Figura 1).

Teniendo todo lo anterior, se realiza el diagnóstico de síndrome de impactación cubital, se decide realizar tratamiento quirúrgico debido a que el tratamiento médico conservador ya había fracasado.

\section{Técnica quirúrgica}

Se intervino quirúrgicamente, bajo bloqueo regional en posición de decúbito supino, con equipo artroscópico completo, previa asepsia y antisepsia, colocación de campos estériles, con el brazo asegurado en torre de tracción (posicionador SPIDER2) mantenida de 10-15 libras durante el procedimiento a través de sujetadores de dedos colocado en el dedo índice y anular, con el codo a $90^{\circ}$ de flexión, la cápsula articular de la muñeca es distendida con $10 \mathrm{~mL}$ de solución salina estéril (Figura 2).

\section{Evaluación artroscópica .11001010}

El artroscopio es introducido en portal 3-4 mediante técnica estándar, la salida de la solución fue obtenida colocando aguja hipodérmica número 18 a través del portal 6R o $6 \mathrm{U}$. El recorrido artroscópico se realizó de rutina desde el portal mediocarpal hasta el portal cubital, visualizando sinovitis con ruptura central del fibrocartílago triangular (Figura 3).
Realizando procedimiento a través de portal 6R, desbridando la ruptura central del fibrocartílago triangular con rasurador mecánico $2.9 \mathrm{~mm}$ hasta dejar bordes estables, con fresa redonda $3.0 \mathrm{~mm}$ (Arthrex) es usada para remover todo el cartílago articular distal del cúbito, así como los quistes subcondrales a través del defecto central ante desbridado del fibrocartílago triangular. Con la misma fresa se realiza el acortamiento del extremo distal del cúbito, con el ayudante que realiza movimiento de rotación de la muñeca desde la supinación hasta la pronación completa, para así realizar una adecuada exposición y resección del cúbito distal mientras se protege la inserción del fibrocartílago triangular en la base del estiloide cubital.

Se realiza sinovectomía, se extraen los cuerpos libres articulares, se realiza hemostasia con ayuda de radiofrecuencia. Al final de la cirugía se obtuvo una varianza cubital de neutral a $-2 \mathrm{~mm}$, se cierran los portales con prolene 0000, y se inmoviliza con férula antebraquiopalmar.

Es valorada en consulta externa a los 10 días posteriores a la cirugía, se retiran puntos, se realiza cambio de férula por molde de yeso de fibra de vidrio, luego se cita a las cuatro semanas de la cirugía, en la cual se retira molde de yeso, paciente aqueja leve dolor en región cubital, se envía a terapia física en casa. 
A las seis semanas postquirúrgicas se envía a la paciente a 10 sesiones de terapia física formal para iniciar ejercicios de fortalecimiento, arcos de movilidad. A las 12 semanas, se encuentra sin dolor, con rangos de movimientos completos, adecuada fuerza de agarre y prensión, con puntuación DASH (The Disabilities of the Arm, Shoulder and Hand Score) favorable, que es un instrumento específico que mide la calidad de vida relacionada con los problemas del miembro superior, EVA de 1/10.

\section{DISCUSIÓN}

El síndrome de impactación cubital puede tratarse mediante una variedad de métodos quirúrgicos y
Figura 2:

Referencia anatómica y equipo básico, para artroscopia de muñeca. $1=$ Portal artroscópico 3-4. 2 = Portal artroscópico 6R. 3 = Estiloide cubital. 4 = Línea articular radiocarpiana.
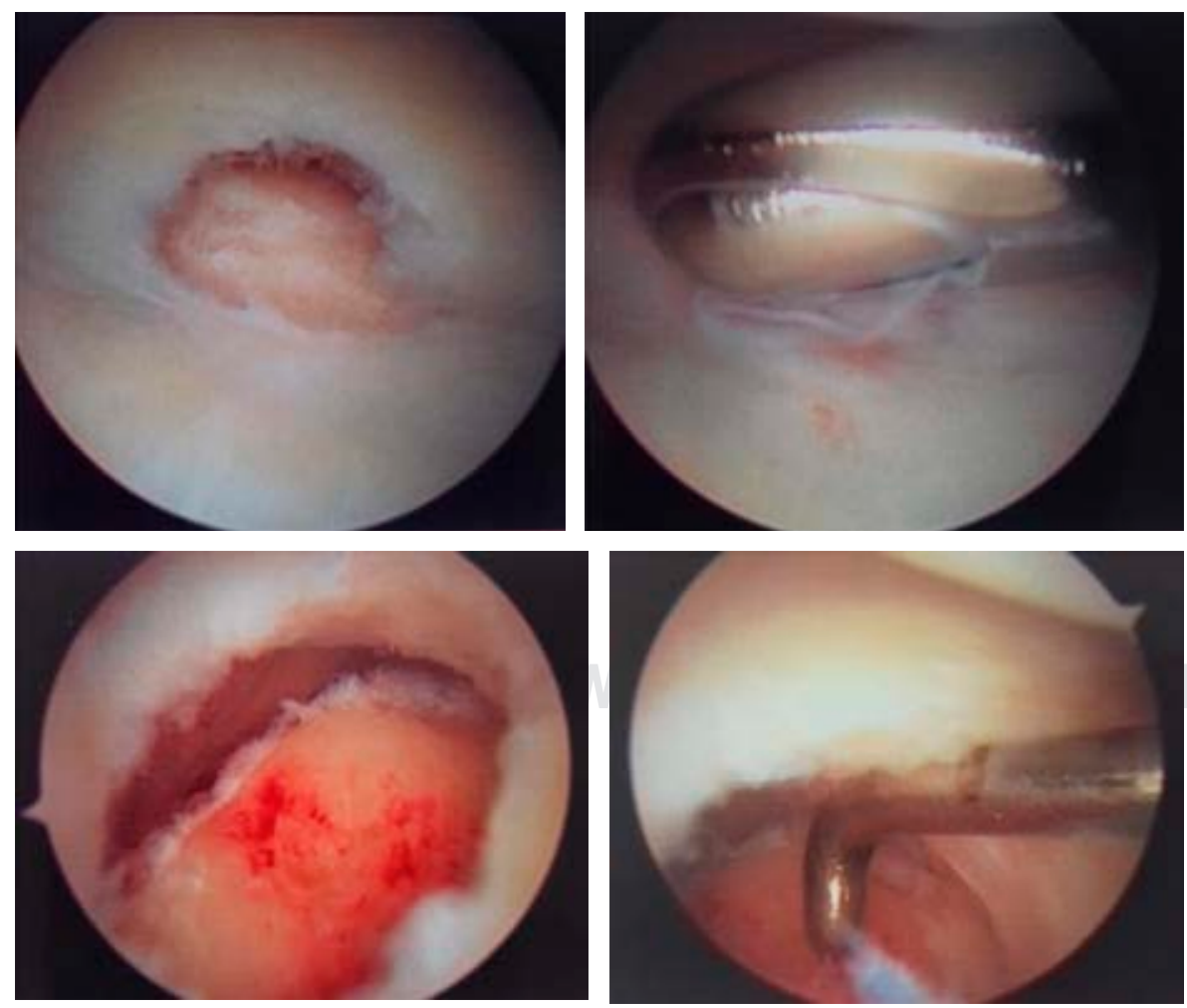

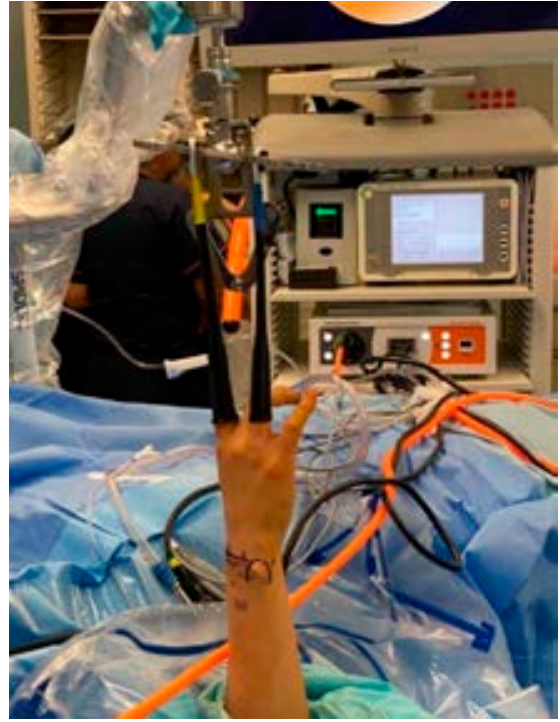

Figura 3:

Evaluación artroscópica del fibrocartílago triangular y realización de procedimiento de Wafer. 
no quirúrgicos, los cuales van desde medicamentos antiinflamatorios, inmovilización e inyecciones de corticosteroides hasta el desbridamiento de CFCT, acortamiento cubital desde las osteotomías y procedimientos artroscópicos de Wafer.

Se ha demostrado que el desbridamiento de los desgarros CFCT sólo en muñecas con varianzas cubitales positivas ha proporcionado resultados incompletos y alivio en hasta $25 \%$ de los pacientes.

Tomaino y Shah en 2001 mostró buenos resultados al combinar desbridamiento del CFCT con el procedimiento artroscópico de Wafer en pacientes que tenían varianzas cubitales positiva. ${ }^{11,12}$

Constantine y colaboradores en 2000 compararon las osteotomías de acortamiento cubital con el procedimiento artroscópico de Wafer, que muestra que ambos fueron eficaces para aliviar el dolor, pero que el procedimiento artroscópico de Wafer no fue complicado por hardware y problemas de pseudoartrosis o no unión ósea. ${ }^{13}$

Beredjiklian y su grupo describieron complicaciones de la artroscopia de muñeca después de tratar a 211 pacientes y encontraron una tasa de complicaciones de $5.2 \%$, que incluyó rigidez, formación de gangliones en el dorso de la muñeca, neuropraxia dorsal cubital sensorial e infección superficial del sitio del portal, así como una quemadura de primer grado con un artroscopio caliente. ${ }^{11,14}$

Kyle D Bickel menciona en su artículo de tratamiento artroscópico del síndrome de impactación cubital que las complicaciones de la artroscopia de muñeca pueden incluir sensibilidad a la cicatriz del portal, tendinitis del compartimento extensor, cicatrización capsular dorsal con flexión y extensión limitadas de la muñeca, lesión de la zona sensorial dorsal radial o cubital, nervios y lesiones cartilaginosas por instrumentación. ${ }^{15}$

En general, la artroscopia es un procedimiento seguro y evita otras complicaciones observadas con procedimiento de acortamiento cubital abierto. El procedimiento artroscópico de Wafer es una buena opción a considerar en pacientes en quienes el tratamiento conservador para el síndrome de impactación cubital ha fracasado, con menos de $3 \mathrm{~mm}$ de varianza cubital positiva (porque más de 2 a $3 \mathrm{~mm}$ es difícil de eliminar artroscópicamente) y ruptura de CFCT. ${ }^{11,16}$

\section{CONCLUSIÓN}

Tratamiento artroscópico del síndrome de impactación cubital con desbridamiento CFCT y resección de Wafer cubital permite resultados eficaces de una sola etapa con excelente alivio del dolor en la mayoría de los pacientes. Se simplifica un segundo procedimiento, exposición quirúrgica extensa y numerosas complicaciones en comparación con el acortamiento cubital mediante osteotomía, lo cual hace de éste el procedimiento de elección para pacientes debidamente seleccionados.

\section{REFERENCIAS}

1. Bernstein MA, Nagle DJ, Martinez A, Stogin JM Jr, Wiedrich TA. A comparison of combined arthroscopic triangular fibrocartilage complex debridement and arthroscopic wafer distal ulna resection versus arthroscopic triangular fibrocartilage complex debridement and ulnar shortening osteotomy for ulnocarpal abutment syndrome. Arthroscopy. 2004; 20 (4): 392-401.

2. Hinzpeter KD. Diagnóstico y tratamiento del dolor cubital de muñeca en el deportista. Rev Med Clin Condes. 2012; 23 (3): 299-312.

3. Barbaric K, Rujevcan G, Labas M, Delimar D, Bicanic G. Ulnar shortening osteotomy after distal radius fracture malunion: review of literature. Open Orthop J. 2015; 9: 98-106.

4. Werner FW, Glisson RR, Murphy DJ, Palmer AK. Force transmission through the distal radioulnar carpal joint: effect of ulnar lengthening and shortening. Handchir Mikrochir Plast Chir. 1986; 18 (5): 304-308.

5. Milch H. Cuff resection of the ulna for malunited Colles' fracture. J Bone Joint Surg Am [Internet]. 1941; 23 (2): 311-313. Available in: https://journals.Iww.com/jbjsjournal/Fulltext/1941/23020/CUFF RESECTION_OF_THE_ULNA_FOR_MALUNITED_COLLES_.9.asp $\bar{x}$

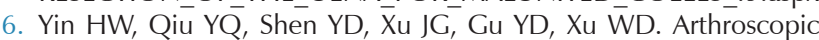
distal metaphyseal ulnar shortening osteotomy for ulnar impaction syndrome: a different technique. J Hand Surg Am. 2013; 38 (11): 2257-2262.

7. Feldon P, Terrono AL, Belsky MR. Wafer distal ulna resection for triangular fibrocartilage tears and/or ulna impaction syndrome. J Hand Surg Am. 1992; 17 (4): 731-737.

8. Wnorowski DC, Palmer AK, Werner FW, Fortino MD. Anatomic and biomechanical analysis of the arthroscopic wafer procedure. Arthroscopy. 1992; 8 (2): 204-212.

9. Carratalá Baixauli V, Lucas García FJ, Sánchez Alepuz E. Dolor en el borde ulnar de la muñeca. Rev Esp Artrosc Cir Articul. 2014; 21 (1): 77-80.

10. Carratalá Baixauli V, Lucas FJ, Calero Ferrandis R, Sánchez Alepuz E. Síndrome de impactación ulnocarpiano, manejo artroscópico. Rev Esp Artrosc Cir Articul. 2014; 21 (1): 28-36.

11. Colantoni J, Chadderdon C, Gaston RG. Arthroscopic wafer procedure for ulnar impaction syndrome. Arthrosc Tech. 2014; 3 (1): e123-e125.

12. Tomaino MM, Shah M. Treatment of ulnar impaction syndrome with the wafer procedure. Am J Orthop (Belle Mead NJ). 2001; 30 (2): 129-133.

13. Constantine KJ, Tomaino MM, Herndon JH, Sotereanos DG. Comparison of ulnar shortening osteotomy and the wafer resection procedure as treatment for ulnar impaction syndrome. J Hand Surg Am. 2000; 25 (1): 55-60.

14. Beredjiklian PK, Bozentka DJ, Leung YL, Monaghan BA. Complications of wrist arthroscopy. J Hand Surg Am. 2004; 29 (3): 406-411.

15. Bickel KD. Arthroscopic treatment of ulnar impaction syndrome. J Hand Surg Am. 2008; 33 (8): 1420-1423.

16. Esplugas M, Aixala Llovet V. Lesiones del complejo del fibrocartílago triangular. Tipos de reparación. Rev Esp Artrosc Cir Articul. 2014; 21 (1): 14-27. 